RUNNING HEAD: THE EMERGENT PRACTICE OF INFANT COMPLIANCE

TITLE: The emergent practice of infant compliance: an exploration in two cultures

Vasudevi Reddy, Katja Liebal and Kerry Hicks

University of Portsmouth, UK

Srujana Jonnalagadda and Beena Chintalapuri

Osmania University, Hyderabad, India

\title{
AUTHOR NOTES:
}

This study was conducted at the Department of Psychology, University of Portsmouth, King Henry Building, King Henry $1^{\text {st }}$ Street, Portsmouth PO1 2DY, UK, and College of Arts and Commerce, Osmania University, Hyderabad 500007, India.

Katja Liebal is now at the Freie Universität Berlin, Cluster of Excellence Languages of Emotion, Department of Education and Psychology, Evolutionary Psychology, Berlin, Germany. Kerry Hicks is now at the Child and Adolescent Unit, New Street Health Centre, Barnsley, UK.

This research was supported in full by a grant from the European Union $6^{\text {th }}$ Framework.

Correspondence should be addressed to Vasudevi Reddy, Department of Psychology, University of Portsmouth, King Henry Building, King Henry $1^{\text {st }}$ Street, Portsmouth PO1 2DY, UK. E-mail:vasu.reddy@port.ac.uk 


\begin{abstract}
Complying with directives is an important indicator of developing cooperativeness and of the awareness of others' intentions for one's own actions. Nonetheless, we know little about the early emergence of compliance. The present longitudinal study explores this phenomenon in naturalistic settings in two cultural groups. Nine middle-class urban families in the UK and thirteen middle-class urban families in India were video-taped at home when their infants were $6.5,8,9.5,11$ and 12.5 months of age. Parental directives were present from 6.5 months, increasing with age in both groups, but with higher frequencies in India at all ages. Most directives were positive requests and communicated from a distance. In both groups two-thirds of the directives involved repetition of content with common routines. Compliance increased gradually with age in frequency but not as a proportion of opportunities to comply. The prevalence, but not the proportion, of compliance was earlier and more frequent in India. In both groups, the rate of change over age in the frequency of parental directives was always steeper than, but strongly related to, the rate of change in the frequency of infant compliance. The emergence of cooperation with requests is situationally-embedded and based on practice.
\end{abstract}

Key words: infancy, awareness of intentions, communication, compliance, socio-cognitive skills, directives 
The emergent practice of infant compliance: an exploration in two cultures

Complying with others' requests is essential to cooperative activity in most aspects of social life. It is also crucial for understanding the developing awareness of others' intentions: responding cooperatively to directives requires some awareness that the other wants you to do something, and what that something is. Infant compliance with requests thus straddles two fields: cooperation with rules and intention awareness. However, although known to be established by the end of the first year, we know little about how early compliance emerges in everyday interactions. Recent evidence showing that infants are sensitive to the intentionality of others' actions by 6 months suggests that the middle of the first year is a crucial period for investigating the origins of compliant responses. The present study is an exploration of infant responses to naturally occurring adult directives in two cultural groups in the second half of the first year.

\section{Studies of Infant Compliance}

Research on compliance has been primarily directed towards understanding the development of self-regulation and the emergence of conscience. Three emphases have hindered direct answers to questions about the emergence of, and social influences on, infant compliance in the first year:

One, there has been a greater focus on compliance with (longer-term) committment (Kochanska \& Aksan, 1995; Kochanska, Aksan \& Koenig, 1995), which does not address the question of how infants begin to comply. Two, most research on responses to directives focuses on the second year or later (e.g., Gralinski \& Kopp, 1993; Kaler \& Kopp, 1990;

Kuczynski, Kochanska, Radke-Yarrow \& Girnius-Brown, 1987; McLaughlin, 1983; Power \& Chapieski, 1986; Schaffer \& Crook, 1980). Although there is evidence that compliance is present by the end of the first year (Kopp, 1982; Stayton, Hogan \& Ainsworth, 1971; 
Campos, Kermoian \& Zumbahlen, 1992), there is only one recent study focusing on earlier infant responses to parental directives (Kochanska, Tjebkes \& Forman 1998). Three, there has been a focus on prohibitions and self-regulated restraint. Thus in the study above, Kochanska and colleagues focused on 8 to 10 month-olds' self-initiated cessation of one specific forbidden action. Complying with positive commands has been seen generally as more challenging than complying with prohibitions since it requires a change of state and the performance of a new action rather than just the cessation of an action. This conclusion may, however, be related to the specific tasks used. The majority of studies use pre-planned 'Do' and 'Don't' tasks in which parents are asked to either prohibit or request the performance of a specific activity (Kochanska, Coy \& Murray, 2001; Kochanska et al., 1998; Stifter et al., 1999; Tamis LeMonda et al., 2007). However, there may be different patterns of response to other everyday commands (Hubley, 1983; Stayton et al., 1971).

\section{Studies of Intention Awareness}

There is a gap between studies of compliance on the one hand, which only study the emergence of compliance towards or after the end of the first year and, on the other, studies of intention awareness, showing that infants are sensitive to the goal-directedness of others' actions from at least 6 months of age (Woodward, 1998; Hamlin, Wynn \& Bloom, 2007). These studies do not address the question of how and when infants start to respond appropriately to others' intentions directed towards the infant's intentional actions, but it seems plausible that around 6 or 7 months of age infants should be sensitive to the intentional meaning in simple requests such as 'You want me to do something' or 'You want me to stop'. Debates about the awareness of communicative intentions have distinguished between directives delivered as physical pressure versus as verbal requests, raising the question of whether compliance emerges first only to perceivable physical requests or also in response to 
distal requests (arguably involving inference about communicative intent) (Tomasello 1999; Tomasello \& Camaioni, 1997; Senju \& Csibra, 2008). The early study of directives and compliance is vital for understanding the developing awareness of intentions.

\section{Social experience and the emergence of compliance}

Interactive experience is known to influence socio-cognitive development (Hofer, Hohenberger, Hauf \& Aschersleben, 2008; Karmiloff-Smith et al, 2010; Rowe \& GoldinMeadow, 2009; Tamis-LeMonda, Bornstein \& Baumwell, 2001). Most studies on compliance have been conducted in Anglo-American cultures. However, in comparison with European families, higher frequencies of directives to children have been reported in some South Asian cultures such as India (Kaertner, Keller, Chaudhary \& Yovsi, in press; Liebal, Reddy, Hicks, Jonnalagadda \& Chintalapuri, 2011; Reddy, 1983). These differences in parental behavior may extend to the onset of directive practices in infancy and may thus present very different situations for the emergence of infant compliance. Indeed, from some perspectives, differences in the frequency of directive engagements could directly affect the emergence of compliance as a skill (Bibok, 2011; Carpendale \& Lewis, 2004; Mundy \& Gomes, 1998) or as part of a conventional routine or script (Nelson, 1981).

Thus the present study explores naturally occurring parental directives and infant responses in two samples in India and the UK, tracing developmental changes in the second half of the first year of infancy.

\section{Method}

This was a cross-cultural, longitudinal study using naturalistic observations. Data were collected in participants' homes over a 6-month period at five age points six weeks apart. In keeping with the principles of micro-genetic research (Lavelli et al, 2005), observations 
began at 6.5 months, just before any reports of infant compliance and around the time when studies of intention awareness are beginning to show a significant convergence of developments and ended at 12.5 months, when studies of compliance suggest that it is wellestablished.

\section{Participant Recruitment and Demographic Details}

Participants for this study were recruited from large urban settings in two countries:

Portsmouth in the UK and Hyderabad in India. In Portsmouth recruitment was done through an all-staff email sent around the university, through posters displayed around the campus and through an advert in a local newspaper. In Hyderabad recruitment was done through advertising on the University campus, and at doctors' surgeries. No infants had any serious health problems or sensory deficits.

In Portsmouth the data from 9 families ( 5 boys) were used for analysis ( 4 further families were omitted as they missed visits at two age points). In Hyderabad data from 13 families (8 boys) were used; 11 families ( 6 boys) participated at all 5 age points and 2 families (both boys) participated from 8 months onwards. The families in both samples were of middle socio-economic status, determined by parents' occupation. They differed, however, in the composition of households, with several of the Indian families living in semi-joint family circumstances and daily contact with neighbors and grandparents.

\section{Ethics issues and Informed Consent}

Ethical approval for the study was obtained according to the normal procedure through the Research Ethics Committee of the Department of Psychology at the University of Portsmouth with Informed Consent obtained from all families.

\section{Procedure}


Infants and the interactions with their parents were videotaped at five age points (Means are given in months and days and SDs in days): 6.5 months $(M=6 ; 22 ; S D=6.4), 8$ months $(M=$ $8 ; 7 ; S D=5.4), 9.5$ months $(M=9 ; 19 ; S D=4.4), 11$ months $(M=11 ; 6 ; S D=5.4)$ and 12.5 months $(M=12 ; 18 ; S D=4.7)$. Each visit lasted approximately 1 hour $(M=65 \mathrm{~min} ; S D=$ 13.6).

Parents were informed that we were studying the development of the infant's social interactions and were asked to do what they normally did during the day. Infants were occasionally left for short periods without a caregiver in the room (Portsmouth: $M=29$ seconds, $S D=55$; Hyderabad: $M=95$ seconds, $S D=103$ ). In both groups between $75 \%$ and $86 \%$ of the visits at each age included an infant mealtime. All infant interactions with their parents and other caregivers (grandparents, maids and familiar relatives) were coded.

\section{Coding of Parental Directives and Infant Compliance}

Table 1 shows the definitions of the variables and codes used to identify directives and the infant's responses. Only the first directive in an episode and the first response by the infant were analyzed rather than eventual responses following repeated persuasions (Mundy et al, 2003).

\section{TABLE 1 ABOUT HERE}

Coder reliability. This was achieved in three stages due to the different languages of the two groups. First, two coders from Portsmouth independently coded $10 \%$ of the data from the Portsmouth sample. Second, reliability was established between the principal Portsmouth coder and the Hyderabad coder using the same data used for the first round of reliability. Third, the first author, who spoke English as well as Telugu, coded 10\% of the Hyderabad data. Coefficients of agreement were calculated for the identification of a) parental directive episodes and b) repeated content. Cohen's kappas $(\kappa)$ were calculated for all other aspects of 
directives and responses, ranging from moderate $(\kappa=.72)$ to excellent $(\kappa=.92)$ levels of agreement (Fleiss, 1981) (see Table 1).

\section{Results and Discussion}

Three main findings emerged from this study, and they are presented below in three sections. First, parental directives were prevalent early, were largely distally communicated, were primarily positive and involved repetitions of content; they increased in frequency with age in both groups but were always higher in Hyderabad than in Portsmouth. Second, in both groups the prevalence of compliance increased gradually with age, with some compliance evident even at 6.5 months in Hyderabad; proportions of compliance were stable over age. Third, in both groups, directive frequency was related to the frequency but not the proportion of compliance; the rate of change in directive frequency was always steeper than the rate of change in frequency of compliance. In sum, directive engagements provided early interactive processes for the emergence of compliance. The small sample sizes demand caution in the interpretation of non-significant differences, but nonetheless suggest some intriguing patterns of similarity and continuity.

\section{Parental directives}

Frequencies per hour: Despite substantial individual variability in frequency, directives were present in all families from the start of the study. About a quarter of all directives at each visit $(M=22.12, S D=5.30)$ were immediately redundant (Table 1$)$ with no group or age differences. These were directives which either accompanied the act (also reported later in development, McLaughlin, 1983), were interrupted by events, or in which the adult intervened to perform the act before the infant had a chance to do it. These immediately redundant directives were excluded from analyses.

\section{FIGURE 1 ABOUT HERE}


In both groups frequencies changed with age, with a significant increase in Portsmouth between 9.5 and 11 months $(z=2.55, p=.011)$ and in Hyderabad between 8 and 9.5 months $(z=2.90, p=.004)$ followed by a decrease $(z=2.90, p=.004)$ and reversal of this decrease $(z=3.11, p=.002)$, (Figure 1). At all ages directives were more frequent in Hyderabad than in Portsmouth (Mann-Whitney at each age: $p$ - values ranged from .006 to .000008). The high frequencies of directives by 12.5 months (one every two minutes in Portsmouth and one every minute in Hyderabad), although still lower than reports of one directive every 9 seconds in the second year (Schaffer \& Crook, 1980) paint a picture of intense parental involvement in infant actions. The high variability between families and cultures is consistent with findings in older age groups (Kaertner et al, in press; Reddy, 1983). Its presence even before verbal discourse comes into play (Dunn \& Munn, 1986), and at what is a crucial period of development for intention-awareness (Woodward, 1998; Baldwin \& Baird, 2002; Karmiloff-Smith et al, 2010) suggests that we cannot make general acultural conclusions about infants' experiences of others' intentional actions directed towards them.

Distal and Physical Directives. Distal directives were significantly more frequent than physical directives at each age and in both groups (Wilcoxon at each age: $p$ - values ranged from to .008 to .012 in Portsmouth and from .001 to .003 in Hyderabad). Parents were, at least in the initial expression of the directive, using the vast majority of their directives as distal communications intended for the infant to respond, perhaps capitalizing on rapid word learning abilities in infants from 6 months (Friederich \& Friedrici, 2011). As a proportion of all directives, distal directives increased from 8 months to 12.5 months in Portsmouth $\left(\chi^{2}(3)=\right.$ $13.53, p=.004$ ) but not in Hyderabad (Table 2). There were no group differences at any age.

\section{TABLE 2 ABOUT HERE}

Positive and Negative Directives. At each age, and in both groups, the frequency of positive directives was substantially higher than the frequency of negative directives (Wilcoxon's at 
each age: Portsmouth $p$ - value of .008; Hyderabad $p$ - value from .001 to .003). Infants' experiences of directives were thus predominantly as requests for positive actions, as in the second year (Schaffer \& Crook, 1980; McLaughlin, 1983). The incidence of negative directives was very low at 6.5 and 8 months, but mean proportions of positive directives between 8 and 12.5 months did not show significant change with age in either group (Table 2). There were no group differences at any age.

Repetition of directive content. In both groups at least two-thirds of all directive episodes were repeats of the same content (Table 2). Overall, there were higher proportions of repeated content in Hyderabad than in Portsmouth (Mann-Whitney $\mathrm{U}(20)=8.0, \mathrm{p}=.001$ ), with no age effects in either group. Such repetition over the course of an hour of requests for specific actions gives infants in both groups considerable practice with these specific directives, forming routines whether temporary or long-term and whether specific to the family or more generally to the culture. Routines allow infants to be more sensitive to the cues and features of common situations in which their actions are required (Nelson, 1981, 2007).

\section{Infant Compliance}

Frequencies per hour and proportions. Compliant responses were present from 6.5 months of age in the majority of infants (with only three infants in Portsmouth showing no compliant responses). In both groups the frequency of compliance was associated with age overall (Friedman: Portsmouth: $\chi^{2}(4)=9.96, p=.041$; Hyderabad $\chi^{2}(4)=13.6, p=.009$ ). Increase between consecutive ages was gradual in both groups, with only one significant increase, at 12.5 months in Hyderabad following a non-significant dip at 11.5 months (Figure 1). Frequencies of compliance were higher in Hyderabad than in Portsmouth at all ages (MannWhitney at each age: $p$ - values ranged from .003 to .000004). As a proportion of opportunities to comply, compliance showed no consistent age or group differences (mean 
proportions ranged between .30 and .37 in Portsmouth and between .35 and .42 in Hyderabad). This is roughly similar to proportions of compliance in the second year of between $30 \%$ and $60 \%$ depending on the method used (Matas, Arend \& Sroufe, 1978; McLaughlin, 1983; Schaffer \& Crook, 1980; Power, McGrath, Hughes \& Manire, 1994). Compliance to Distal Directives. Proportions of compliance to distal directives showed no overall effect of age in either group (mean proportions between 8 and 12.5 months ranged from .27 to .39 in Portsmouth and from .37 to .42 in Hyderabad). Frequencies of physical directives were too low for calculating proportions of compliance.

Compliance to Positive Directives. Proportions of compliance to positive directives showed no overall effect of age in either group (mean proportions between 8 and 12.5 months ranged from .33 to .36 in Portsmouth and from .36 to .46 in Hyderabad). Frequencies of negative directives were too low for calculating proportions of compliance.

\section{TABLE 3 ABOUT HERE}

Compliance at 6.5 and 8.0 months. Since infant responses to directives before 9.5 months are the least expected and the least known, Table 3 shows examples of directives and infant responses at 6.5 and 8 months from each group. At 6.5 months compliance was mostly evident to requests for Attention, to requests to Take or Hold an object and to requests to Eat. The affordances of accompanying prompts (e.g., proffered object, spoon or point) might be sufficient to elicit the required action, but also socially frame the infant's action by linking the act, the affordances and the adult's utterances and expressions. Infant compliant acts were not automatic or reflexive but revealed an integration of the response with awareness of the directive act (Exs. 2, 6, 7, 9, 10). Table 3 illustrates different kinds of routines: games (rolling the ball, or the 'head-hitting' game in Hyderabad), standard uses of specific toys (pulling X on a string, opening part of a toy), new motor skills (sitting, standing), gestures (waving, 
clapping, kissing, the 'come' gesture in Hyderabad), and location requests (Show where's Aunty, Daddy, the fan). By 8 months, compliance occurs to a broader range of content, with several cases in Hyderabad of compliance with requests for conventional gestures (Ex. 20), conventional practices (Ex. 18), and family specific 'tricks' (Exs. 16 and 19). The specific ways in which compliance is differentially supported by contextualization remain to be explored in future research and tested within larger samples.

\section{Parental directive frequency and infant compliance.}

We used hierarchical linear modeling software (HLM 6.06) to explore the relationship between directive frequency and infant compliance at a given age. A two-level model was created with multiple observations of directives and compliance at level 1 , nested within families at level 2. At level 2, culture was included as a random effect categorical variable. Directive frequency was overall strongly related to infant compliance frequency $(\gamma=.32$, $t(20)=7.01, p<.001)$, with no difference between the two groups in this relation $(\gamma=.04$, $t(20)=1.31, p=.21)$. However, there was no relation between directive frequency and comply proportions overall $(\gamma=.35, \mathrm{t}(20)=1.07, p=.30)$, nor any difference between the groups in the relation $(\gamma=-.16, \mathrm{t}(20)=-.93, p=.35)$. We calculated slopes (the rate of change) over the 5 age-points for frequencies of directives and of compliance (with linear trendlines fitted). The mean slope for directive frequency (Portsmouth: $M=5.11 \mathrm{x}, S D=$ 2.53; Hyderabad: $M=7.68 \mathrm{x}, S D=6.16$ ) was steeper in both groups (Portsmouth: Wilcoxon's: $z=2.67, p=.008$; Hyderabad: Wilcoxon's: $z=2.85, p=.004$ ) than the mean slope for compliance frequency (Portsmouth: $M=1.60 \mathrm{x}, S D=1.70$; Hyderabad: $M=2.98 \mathrm{x}$, $S D=3.75)$. Thus, parental changes increased more rapidly than infant changes, suggesting that parental changes serve to 'pull' infant responses or reflect accommodation to infant changes - or, most likely, do both these things - with the proportion of compliance remaining steady. 


\section{General Discussion}

Parental directives are socio-cultural practices influenced by cultural contexts and serving to draw infants into participation in directive-compliance routines. In both groups, directives were largely expressed without physical pressure in the initial instance and were predominantly requests for positive actions. These results support, and extend to earlier ages, the emphasis on a 'bias toward social behavior' (Kopp, 1982) and shared interactive positivity as mediators of compliance (Kochanska, Forman, Aksan \& Dunbar, 2005; Kochanska et al, 1999). Parents are not just regulating infants' behavior in terms of its negative or pragmatic potential, but predominantly in terms of its potential for participating in cultural practices and interactive enjoyment. Early compliance to positive directives in this study supports and extends the finding of higher 'situational' (short-term) compliance to positive directives than to negative directives in the second year (Kochanska et al, 2001) but argues against the idea that positive commands are more challenging than negative ones. In fact, positive directives may afford more compliance than negative ones since they are less likely to involve motivational conflict, more likely to be part of familiar family routines, and often likely to be accompanied by perceptual prompts.

Katherine Nelson in 1981 put forward three challenges (to what she termed static structural models of socio-cognitive development), the first of which was that of finding a way to describe the acquisition of knowledge within participatory frameworks rather than as representations of abstract categorical knowledge. The present data go some way towards meeting this challenge by showing that parental directives are prevalent very early in infancy, are often redundant, differ in frequency between cultures and have frequently repeated content and routines thus offering infants practice and contextual affordances for specific actions. The directive intentions of even distal directives, may therefore be perceived within 
routines and contexts rather than having to be inferred. It might be more fruitful to conceptualise the understanding of requesting intentions as something that does not just occur 'in the head' but is always embedded in situational and material practices (Sinha, 1982). Views of the 'extended mind' - for instance, when one's diary is a part of one's remembering, or the layout of a screen contains one's knowledge of how to use a programme - could be useful for theorising the understanding of directive intentions (see also Nelson, 2007).

The gradual increase over age in frequencies of compliance in both groups, with earlier prevalence of both directives and compliance in Hyderabad, suggests that compliance develops as a practice with infants being drawn into recognition of the communicative intentions in directives addressed to them. As with understanding 'reference' (Churcher \& Scaife, 1981), the awareness of others' requesting intentions cannot be fixed to a single time point: compliance emerges specific to content and its emergence is imperceptible within the context of routine cooperative engagements. Even before 6 months of age, parents seek and infants respond with cooperation in non-verbal engagements (Lock, 1984; Trevarthen \& Hubley, 1978). Shotter (1983) argues that we always act into environmental stimuli, our response enlarging, transforming and reconstituting that which we respond to. With their responses in these directive engagements infants may be seen similarly as acting into adult directives, as adults are acting into infant's compliant responses. Directing and complying may thus need to be re-defined as continuous, emergent and mutually enlarging rather than categorical and separate phenomena.

Parental directives may be creating a genuine 'response space' for infants in a similar way to the 'symbolic space' created by parents who, instead of picking up their infants without ado, offer a pick up invitation and wait for a possible response before fulfilling the action 
(Service, 1984). It could be argued that development only occurs within such problem spaces (Leavens, Hopkins \& Bard, 2005) and that the high frequencies of directives and repeated content in these early months (particularly in Hyderabad) are actually constitutive of the development of compliance. Infants are being guided not only for participating in specific actions (Rogoff, 1993) but also for participation itself. 


\section{References}

Bibok, M. (2011). Re-conceptualizing joint attention as social skills: a microgenetic analysis of early infant communication. $\mathrm{PhD}$ Thesis, Simon Fraser University.

Campos, J. J., Kermoian, R., Zumbahlen, M. R. (1992). Socio-emotional transformations in the family system following infant crawling onset. In N. Eisenberg \& R. A, Fabes (Eds.) Emotion and its regulation in early development (pp. 25-40). San Francisco: Jossey-Bass. doi:10.1002/cd.23219925504

Carpendale, J. \& Lewis, C. (2004). Constructing an understanding of mind: the development of children's social understanding within social interaction. Behavioral and Brain Sciences, 27, 79-151. doi:10.1017/S0140525X04000032

Churcher, J. \& Scaife, M. (1981). How infants see the point. In G. Butterworth \& P. Light (Eds.) Social Cognition: Studies in the development of understanding. Brighton: Harvester Press.

Csibra, G. (2010). Recognizing communicative intentions in infancy. Mind and Language, 25 (2), 141-168. doi:10.1111/j.1468-0017.2009.01384.x

Dunn, J. \& Munn, P. (1986). Siblings and the development of prosocial behaviour. International Journal of Behavioral Development, 9 (3), 265-284. http://ibd.sagepub.com/content/9/3/265.full.pdf+html

Fleiss, J. (1981). Statistical methods for rates and proportions. New York: John Wiley \& Sons. http://eu.wiley.com/WileyCDA/Section/index.html

Friedrich, M. \& Friederici, A. (2011) Word-learning in 6-month-olds: Fast encoding-weak retention. Journal of Cognitive Neuroscience, 23 (11), 3228-3240. $\underline{\text { doi:10.1162/jocn a } 00002}$

Gralinski, H. J., \& Kopp, C. B. (1993). Everyday rules for behaviour: mothers' requests to young children. Developmental Psychology, 29, 573-584. doi:10.1037//0012$\underline{1649.29 .3 .573}$ 
Hamlin, J.K., Wynn, K., \& Bloom, P. (2007). Social evaluation by preverbal infants. Nature, 450, 557-560. doi:10.1038/nature06288

Hofer, T., Hohenberger, A., Hauf, P., \& Aschersleben, G. (2008) The link between maternal interactive style and infant action understanding. Infant Behavior and Development, 31, 115-126. doi:10.1016/j.infbeh.2007.07.003

Hubley, P. (1983). The development of cooperative action in infants. PhD Thesis, University of Edinburgh.

Kaler, S., \& Kopp, C. (1990). Compliance and comprehension in very young toddlers. Child Development, 61, 1997-2003. doi:10.1111/j.1467-8624.1990.tb03581.x

Karmiloff-Smith, A., Aschersleben, G., de Schonen, S., Elsabbagh, M., Hohenberger, A., \& Serres, J. (2010). Constraints on the timing of infant cognitive change: domain-specific or domain-general? European Journal of Developmental Science, 4 (1), 31-45. http://www.v-r.de/en/magazine-0-0/european journal of developmental science500057/\#section ausfuhrliche informationen

Kochanska, G. \& Aksan, N. (1995). Mother-child mutually positive affect, the quality of child compliance to requests and prohibitions and maternal control as correlates of early internalisation. Child Development, 66, 236-254. doi:10.2307/1131203

Kochanska, G., Aksan, N., \& Koenig, A. (1995) A longitudinal study of the roots of preschoolers' conscience: committed compliance and emerging internalization. Child Development, 66 (6), 1752-1769. doi:10.1111/j.1467-8624.1995.tb00963.x

Kochanska, G., Coy, K.C., \& Murray, K. T. (2001). The development of self-regulation in the first four years of life. Child Development, 72 (4), 1091-1111. doi:10.1111/1467$\underline{8624.00336}$

Kochanska, G., Forman, D., \& Aksan, N., \& Dunbar, S. (2005). Pathways to conscience: early mother-child mutually responsive orientation and children's moral emotion, 
conduct and cognition. Journal of Child Psychology and Psychiatry, 46, (1), 19-34. doi:10.1111/j.1469-7610.2004.00348.x

Kochanska, G., Tjebkes, T., L., \& Forman, D. R. (1998). Children's emerging regulation of conduct: restraint, compliance and internalisation from infancy to the second year. Child Development, 69 (5), 1378-1389. doi:10.2307/1132272

Kopp, C. (1982). Antecedents of self-regulation: A developmental perspective. Developmental Psychology, 18, 199-214. doi:10.1037//0012-1649.18.2.199

Kuczynski, L., Kochanska, G., Radke-Yarrow, M., \& Girnius-Brown, O. (1987). A developmental interpretation of young children's noncompliance. Developmental Psychology, 23(6), 799. doi:10.1037//0012-1649.23.6.799

Lavelli, M., Pantoja, A. P. F., Hsu, H., Messinger, D., \& Fogel, A. (2005). Using microgenetic designs to study change processes. In D. Teti (Ed.), Handbook of Research Methods in Developmental Science (pp. 40-65). Oxford: Blackwell. doi:10.1002/9780470756676.ch3

Leavens, D., Hopkins, W., \& Bard, K. (2005). Understanding the point of chimpanzee pointing: Epigenesis and ecological validity. Current Directions in Psychological Science, 14, 185-189. doi:10.1111/i.0963-7214.2005.00361.x

Liebal, K., Reddy, V., Hicks, K., Jonnalagadda, S., \& Chintalapuri, B. (2011). Socialization goals and parental directives in infancy: The theory and the practice. Journal of Cognitive Education and Psychology, 10 (1), 113-131. doi:10.1891/1945-8959.10.1.113

Lock, A. (1984). The emergence of language: on being picked up. In A. Lock \& E. Fisher (Eds.) Language Development. Kent: Croom Helm Ltd. http://isbndb.com/d/publisher/croom helm ltd.html 
Matas, L., Arend, R., \& Sroufe, A. (1978). Continuity of adaptation in the second year: the relationship between quality of attachment and later competence. Child Development, 49, 547-556. doi:10.1111/i.1467-8624.1978.tb02354.x

McLaughlin, B. (1983) Child compliance to parental control techniques. Developmental Psychology, 19 (5), 667-673. doi:10.1037//0012-1649.19.5.667

Mundy, P., Delgado, C., Block, J., Venezia, M., Hogan, A., \& Seibert, J. (2003). A manual for the abridged early social communication scales (ESCS). Unpublished manuscript, University of Miami, Florida.

Mundy, P., \& Gomes, A. (1998). Individual differences in joint attention skill development in the second year. Infant behavior and Development, 21(3) 469-482. doi:10.1016/S0163$\underline{6383(98) 90020-0}$

Nelson, K. (1981). Social cognition in a script framework. In J. Flavell \& L. Ross (Eds.) Social cognitive development: frontiers and possible futures. Cambridge: CUP. www.cambridge.org/9780521281560

Nelson, K (2007) Young minds in social worlds. Cambridge, MA: Harvard University Press. www.hup.harvard.edu/catalog.php?isbn $=9780674034860$

Perner, J., Ruffman, T., \& Leekam, S. (1994). Theory of mind is contagious: you catch it from your sibs. Child Development, 65 (4), 1228-1238. doi:10.1111/j.14678624.1994.tb00814.x

Power, T., \& Chapieski, M.L. (1986) Childrearing and impulse control in toddlers: A naturalistic investigation. Developmental Psychology, 22 (2), 271-275. doi:10.1037//0012-1649.22.2.271

Power, T., McGrath, Hughes., \& Manire, (1994). Compliance and self-assertion: young children's responses to mothers and fathers. Developmental psychology, 30 (6), 980989. doi:10.1037//0012-1649.30.6.980 
Reddy, V. (1983). Responsiveness and compliance in parents' interactions with 7 year-olds in India and Scotland. PhD thesis, University of Edinburgh.

Rogoff, B. (1993) Children's guided participation and participatory appropriation in sociocultural activity. In R.H. Wozniak \& K.W. Fischer (Eds.) Development in context; Acting and thinking in specific environments (pp. 121-1530. Hillsdale, NJ: Lawrence Erlbaum Associates. http://isbndb.com/d/publisher/lawrence_erlbaum_associates.html

Rowe, M., \& Goldin-Meadow, S. (2009). Differences in early gesture explain SES disparities in child vocabulary size at school entry. Science, 323 (5916), 951-953. doi:10.1126/science.1167025

Schaffer, R., \& Crook, C. (1980). Child compliance and maternal control strategies. Developmental Psychology, 16 (1), 54-61. doi:10.1037/0012-1649.16.1.54

Senju, A. \& Csibra, G. (2008). Gaze following in human infants depends on communicative signals. Current Biology, 18(9), 668-671. doi:10.1016/i.cub.2008.03.059

Service, V. (1984). Maternal styles and communicative development. In A. Lock \& E. Fisher (Eds.) Language Development. Kent: Croom Helm Ltd. http://isbndb.com/d/publisher/croom_helm_ltd.html

Shotter, J. (1983). 'Duality of structure' and 'intentionality' in Ecological Psychology. Journal for the Theory of Social Behavior, 13(1), 19-43. doi:10.1111/j.14685914.1983.tb00460.x

Sinha, C. (1982). Negotiating boundaries: Psychology, biology and society. In S. Rose (Ed.) Towards a liberating biology. London: Allison and Busby. www.allisonandbusby.com

Stayton, D., Hogan, R., \& Ainsworth, M. D. S. (1971). Infant obedience and maternal behaviour: the origins of socialisation reconsidered. Child Development, 42, 10571069. doi:10.2307/1127792 
Stifter, C., Spinrad, T. L., \& Braungart-Rieker, J. M. (1999). Toward a developmental model of child compliance: The role of emotion regulation in infancy. Child Development, 70 (1), 21-32. doi:10.1111/1467-8624.00003

Tamis LeMonda, K., Adolph, K., Lobo, S., Karasik, L., Ishak, S., \& Dimitropoulou, K. (2007). When infants take mothers' advice: 18 month-olds integrate perceptual and social information to guide motor action. Developmental Psychology, 44 (3), 734-746. doi:10.1037/0012-1649.44.3.734

Tamis-LeMonda, C., Bornstein, M., \& Baumwell, L. (2001). Maternal responsiveness and children's achievement of language milestones. Child Development, 72 (3), 748-767. doi:10.1111/1467-8624.00313

Tomasello, M. (1999). Having intentions, understanding intentions and understanding communicative intentions. In P. Zelazo, J. Astington \& D. Olson (Eds.) Developing Theories of Intention (pp. 62-76). Mahwah, NJ: Lawrence Erlbaum Association. http://isbndb.com/d/publisher/lawrence erlbaum associates.html/

Tomasello, M. \& Camaioni, L. (1997). A comparison of the gestural communication of apes and human infants. Human Development, 40 (1), 7-24. doi:10.1159/000278540

Trevarthen, C. \& Hubley, P. (1978). Secondary intersubjectivity: confidence, confiding an acts of meaning. In A. Lock (Ed.) Action, gesture and symbol: the emergence of language. London: Academic Press. $\underline{\text { www.elsevierdirect.com/brochures/academicpress }}$

Woodward, A. (1998). Infants selectively encode the goal of an actor's reach. Cognition, 69, 1-34. doi:10.1016/S0010-0277(98)00058-4 
Table 1 Coding of Parental Directives and Infant Responses

\begin{tabular}{|c|c|}
\hline & 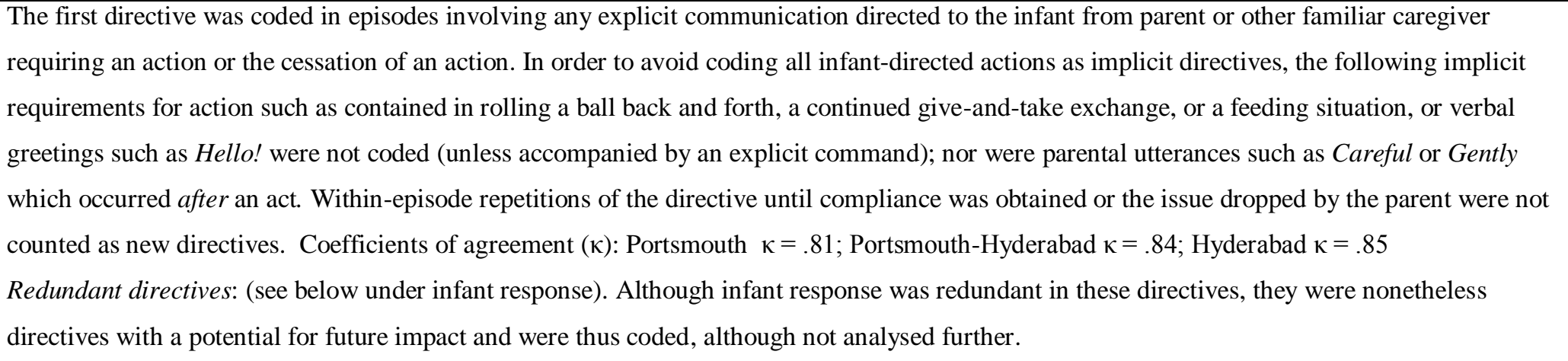 \\
\hline $\boldsymbol{n}$ & $\begin{array}{l}\text { Distal: Any directive which included explicit verbal commands or prohibitions or (provided the context specified the content of the directive) } \\
\text { vocalisations such as aaaa aaaa aaa, with or without a demonstration or gesture, but did not include any physical component (see below). } \\
\text { Physical: Any directive which involved a physical action such as moving the infant's hand or body to help or enforce compliance. For example, } \\
\text { the parent says Press the button and moves the infant's hand part way towards the toy, or the parent while feeding with a spoon says Open... and } \\
\text { pushes the spoon against the infant's mouth to urge the infant to open it. } \\
\text { Portsmouth } \kappa=.82 \text {; Portsmouth-Hyderabad } \kappa=.73 \text {; Hyderabad } \kappa=.84\end{array}$ \\
\hline уре & $\begin{array}{l}\text { Positive: This category included any directive which sought the performance of a specific action, e.g., Press this one or you try it. It also included } \\
\text { directives in which a positive request was immediately followed by a request for cessation of another action e.g., Take this one, don't touch that. } \\
\text { Although initially coded separately, these types of directive were collapsed into one because of low frequencies of the latter type. } \\
\text { Negative: This category included any directive which sought the cessation of a specific action, e.g., No, don't go or Not in your mouth. It also } \\
\text { included directives in which a request for cessation was immediately followed by with a request for a specific action e.g., Don't go there, play with } \\
\text { this. Although initially coded separately, these types of directive were collapsed into one because of low frequencies of the latter type. } \\
\text { Portsmouth } \kappa=.82 ; \text { Portsmouth-Hyderabad } \kappa=.92 ; \text { Hyderabad } \kappa=.87\end{array}$ \\
\hline
\end{tabular}

Repeated content Directives requesting or prohibiting the 'same' actions were often repeated in several episodes during the visit. The number of actions which were repeated in two or more episodes (in the same visit) was divided by the total number of directives in that visit to yield the proportion of repeated content. Actions considered to be the same involved similar levels of skill or effort: requests to 'give' a spoon or a hairbrush were seen as the 
same, while requests to 'fetch' were seen as a different action; requests to do a folded hands gesture for prayer was counted as a different action from being asked to wave 'bye', but being asked to "Show where's Daddy" was seen as being the same as being asked to "Show where's Lily (the $\operatorname{dog}) " \kappa=.86$ started doing it) or if the speaker performed the action themselves immediately after the directive (e.g., the parent says Don't put that in your mouth before the child has a chance to respond) or if an intervening event disallowed the infant's response (e.g., a visitor enters and everyone forgets about the directive) then the directive was coded as immediately redundant.

Comply: When the infant carries out the action specified in the directive; no judgments of intentionality were involved.

Non-comply: This was a category constructed of four types of other coded responses, subsequently collapsed into one category because no age related patterns or group differences were present. The four sub-types were: Part Comply: When the infant started, but did not complete, the specified action. Hesitation: When the infant hesitated briefly on hearing the directive but did not perform the specified action. No Change in response: When the infant did not respond at all to the directive - with attention or hesitation or action. Other non-comply: When the infant did not comply but appeared to be playful or defiant.

Portsmouth $\kappa=.78$; Portsmouth-Hyderabad $\kappa=.72 ;$ Hyderabad $\kappa=.79$ 


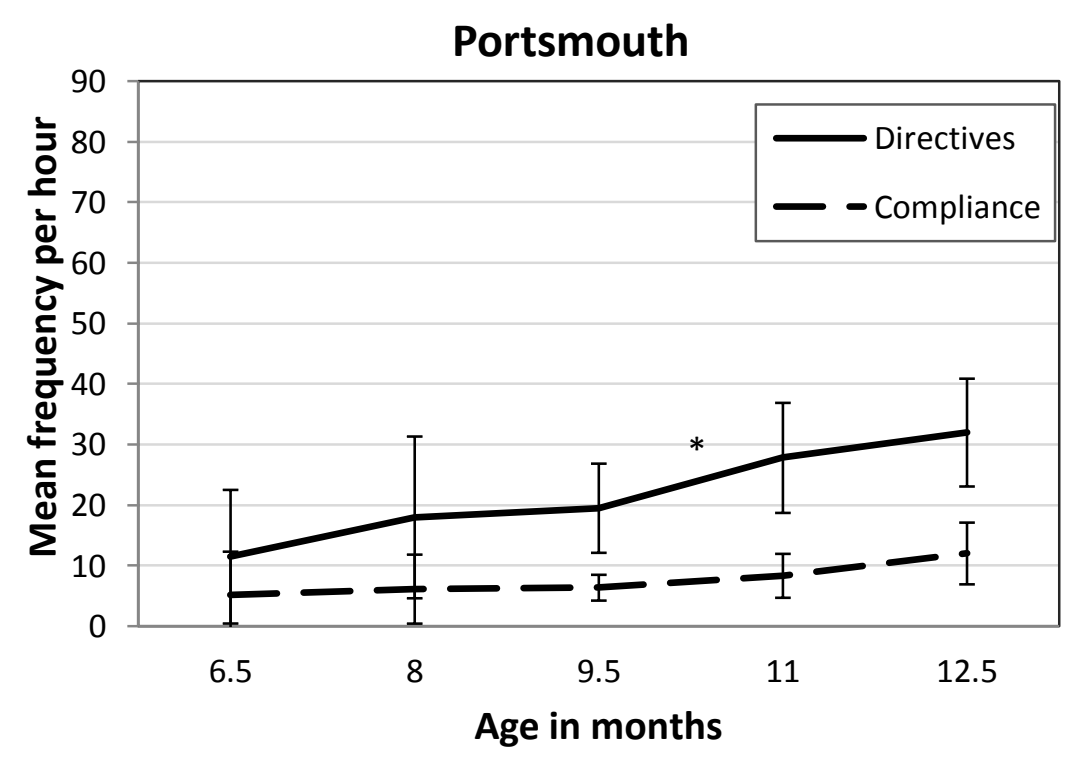

Overall age effects (Friedman's): Directives $\chi^{2}(4)=16.89, p=$ .002 ; Compliance $\chi^{2}(4)=9.96, p=.041$; Differences between consecutive ages (Wilcoxon's): Directives: 9.5 to 11 months: $z$ $=2.55, p=.011$; Compliance: all n.s.

\section{Hyderabad}

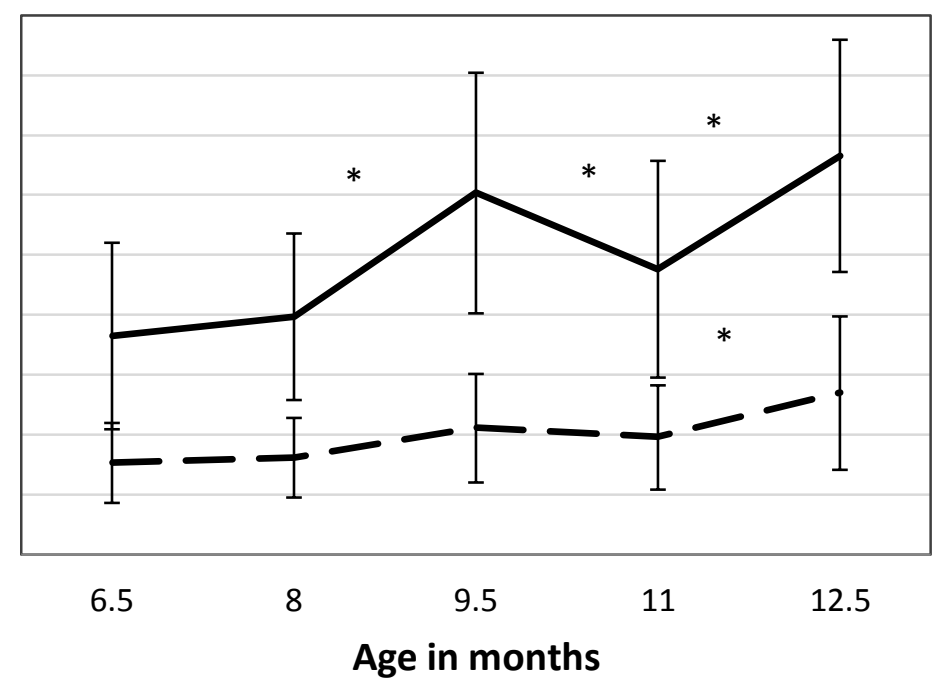

Overall age effects (Friedman's): Directives: $\chi^{2}(4)=19.78, p=$ .001; Compliance: $\chi^{2}(4)=13.6, p=.009$; Differences between consecutive ages (Wilcoxon's): Directives: 8 to 9.5 months: $z=$ $2.90, p=.004 ; 9.5$ to 11 months: $z=2.90, p=.004 ; 11$ to 12.5 months: $z=3.11, p=.002$; Compliance: 11 to 12.5 months: $z=$ $2.13, p=.033$.

NOTE: * refers to a probability value $<.05$ for the difference between adjacent levels

Figure 1. Parental Directives and Infant Compliance: Mean Frequency per hour over age 
Table 2. Proportions (Mean, SD) of Distal Directives, Positive Directives, and Repeated Content

\begin{tabular}{|c|c|c|c|c|c|c|c|c|c|c|c|c|}
\hline & \multicolumn{4}{|c|}{ Proportion Distal } & \multicolumn{4}{|c|}{ Proportion Positive } & \multicolumn{4}{|c|}{ Proportion Repeated Content } \\
\hline & \multicolumn{2}{|c|}{ Portsmouth } & \multicolumn{2}{|c|}{ Hyderabad } & \multicolumn{2}{|c|}{ Portsmouth } & \multicolumn{2}{|c|}{ Hyderabad } & \multicolumn{2}{|c|}{ Portsmouth } & \multicolumn{2}{|c|}{ Hyderabad } \\
\hline & $M^{2}$ & $S D$ & $M$ & $S D$ & $M$ & $S D$ & $M$ & $S D$ & $M$ & $S D$ & $M$ & $S D$ \\
\hline 6.5 & $--^{1}$ & -- & .82 & .11 & $-{ }^{1}$ & -- & .91 & .10 & $-{ }^{1}$ & -- & .79 & .08 \\
\hline 8.0 & .77 & .21 & .86 & .07 & .91 & .12 & .90 & .11 & .66 & .22 & .77 & .15 \\
\hline 9.5 & .84 & .14 & .87 & .08 & .85 & .08 & .86 & .09 & .65 & .16 & .84 & .06 \\
\hline 11.0 & .87 & .05 & .91 & .05 & .84 & .12 & .92 & .10 & .65 & .14 & .74 & .10 \\
\hline 12.5 & .94 & .06 & .90 & .04 & .83 & .14 & .92 & .04 & .70 & .14 & .77 & .07 \\
\hline
\end{tabular}

\footnotetext{
${ }^{1}$ Frequencies of directives too low for calculation of proportions
}

Overall age effects (Friedman's): Proportion Distal: Portsmouth $\chi^{2}(3)=13.53, p=.004$; Hyderabad: n.s.; Proportion Positive: Portsmouth: n.s.;

Hyderabad, n.s.; Proportion Repeated content: Portsmouth: n.s.; Hyderabad: n.s. Group differences (Mann-Whitney): Proportion Distal: n.s.; Proportion Positive: n.s.; Proportion Repeated Content: $\mathrm{U}(20)=8.0, \mathrm{p}=.001$ 
Table 3. Examples of compliance at 6.5 and 8 months

\begin{tabular}{|c|c|c|}
\hline Age & Portsr & Hyder \\
\hline $6.5 \mathrm{~m}$ & $\begin{array}{l}\text { 1. AC: M taps a book: That's horsy look. Inf looking around the room, M } \\
\text { tapping the book again. Inf looks at the picture M is tapping. } \\
\text { 2. JE: Inf turns towards the TV screen. M: What're you after now? Inf turns } \\
\text { away to something else. M: You can see your reflection, can't you? } \\
\text { tapping the screen, Look at yourself. Inf turns to the TV, looks, then turns } \\
\text { to M's face, smiles, and looks back at the TV briefly, then away. } \\
\text { JJ: After a period of playing with a toy telephone, with M demonstrating } \\
\text { the dial, Inf turns to a different toy; M turns the dial again saying What } \\
\text { you were trying to do this morning wasn't it? Inf looks back at the toy } \\
\text { phone. M: You do it. Inf looks, then leans forward and turns the dial. M: } \\
\text { That's it! Good boy. } \\
\text { LC: Inf opens his mouth as M nears with another spoonful, then shuts it } \\
\text { and turns to look at something else. M: Open.. waiting. Inf turns back to it } \\
\text { and opens his mouth. } \\
\text { JE: M demonstrating pulling toy cow with string Can you pull the cow } \\
\text { 5ooches for the string. M: That's it. Inf pulls cow. M: That's a } \\
\text { 4. patting his back. }\end{array}$ & $\begin{array}{l}\text { 6. MA: Inf lying on her back on the bed; M holding her hands in her own, but not pulling her up, says } \\
\text { Get up, come on, come oooon, waiting; Inf makes fussy sounds appearing to want M to pick her up; } \\
\text { M says again come oon looking at her but doesn't pull her up, just waits. Inf stops fussing, looking } \\
\text { at M and starts to strain her neck lifting her head up; M then helps pull her up. } \\
\text { 7. KA: M picks up Inf, asking: Where's Aunty? Aunty? Aunty? F speaks (inaudible). Inf turns to look } \\
\text { at F. M says Show where's Aunty? F repeats the same. Inf turns his head towards the doorway where } \\
\text { Au is standing, smiles broadly on seeing her, and raises both arms briefly. F and M silent, smiling as } \\
\text { they watch Inf look at Au. } \\
\text { SA: M holds Inf upright, then moves her nearer to and facing the wall. M: Hold this. Hold the wall. } \\
\text { Here. Inf looks at the wall, reaches out and puts her palm against it. M: Yes, hold it. Hold it, lets go } \\
\text { as Inf moves her other hand to the wall. } \\
\text { 9. SHS: M holding Inf standing on her lap, Sit down sit down sit down. Sit. Sit. Sit down. Inf looking } \\
\text { to present her cheek to Inf. Inf turns and looks at M's cheek as M repeats Kiss me love. Inf bends } \\
\text { around, doesn't react (not clear whether he has heard). M: Hey, do it, sit and bends to look into Inf's } \\
\text { face. Inf looks at M. M: more softly, Sit sit. Inf bends his knees slowly and sits. } \\
\text { 10. SHS: M carrying Inf who is looking elsewhere: Give me a kiss love, kiss me love... turning her face } \\
\text { 9. }\end{array}$ \\
\hline
\end{tabular}




\section{EM: M carrying Inf who is playing with a stick. M takes the stick away} saying Thank you and, as Inf looks around the room asks Can Mummy have a kiss? Inf turns into M's shoulder and hugs her tightly. All laugh and M says That's nice!

12. JE: Trying to stop I from blowing a raspberry, M: Can you give me a kiss please? Inf looks at the camera, then at M's face, making a lip smacking sound. M: That's it, clever boy!

13. JM: Inf playing with a long sound-making stick, mouthing it. M suggests You gonna wave? Gonna wave with it? waving her own hand in front of Inf's face. Inf looks up at $\mathrm{M}$, smiles and waves her own hand. M waves her own hand vigorously again. Inf looks at M's face then at the toy and waves the toy. M approves: Shake it, that's it.

14. EH: Inf and M at musical toy. M: Ready and opens part of toy. Inf watches. M waits, looking at Inf who is just watching the toy: Can you do $i t$ ? Inf reaches out and does it.

15. AC: Inf hitting a large ball at his side with left arm. M: Are you playing the rolling game? Inf continues hitting the ball with left arm. M: Are you going to roll it to me now? Inf leans whole body to the side, getting arm
16. KA: M, carrying Inf, says How does Thatha cough? Show how Thatha coughs and coughs herself. Inf, although attending to something else, immediately makes an odd vocal coughing sound without looking at M. M laughs and says Yes.

17. SU: M, having walked Inf to the mat with her fingers in his palms, continues to stay that way and says Sit, Mishu, sit, sit and waits. Inf looks up at her. She nods and repeats Sit. He then starts to bend his knees and sit down. She breathes out Oooph in sympathy with his effort.

18. PO: Inf standing up looking down at something, with M lightly holding him. M says Do 'dee', do 'dee' (a head hitting game) moving her face slightly forward but head still upright and waiting (the heads have to be inclined forward to hit foreheads together). Inf looks up at her face then inclines his head forward. $\mathrm{M}$ inclines her head and they hit gently. (This is a regular game among many families).

19. PR: Inf lying on floor on his stomach. M sitting on floor nearby. Au asks Inf Where's Baba? Where's Baba? Baba? (i.e., poster of a Saint) M repeats She's asking show where Baba Thatha is, Show where's Baba Thatha... Inf turns from Au to M, smiling and lifts one arm upwards in an open palm 'point', and still smiling, glances at $\mathrm{V}$ and $\mathrm{Au}$ (reported as a regular routine, even in the absence of the poster).

20. SHA: Inf lying on his stomach looking at toys. M sitting at a distance: Here. Inf turns to look at her. M: Say dai (baby word for come), dai, dai, dai, dai, love, say dai dai, demonstrating a conventional 
The emergent practice of infant compliance 28

\begin{tabular}{|l|l|c|}
\hline behind ball and pushes (it rolls towards M). M: Yaayy!! clapping. & $\begin{array}{c}\text { 'Come' gesture, opening and shutting her hand. Inf turns to look at M, and starts to open and shut } \\
\text { his palm, saying aaaai, aaa. Both repeat it for two seconds then Inf turns back to the toys. }\end{array}$ \\
\end{tabular}

Note: $\mathrm{M}=$ Mother; $\mathrm{F}=$ Father; Inf = Infant; $\mathrm{Au}=\mathrm{Aunt} ; \mathrm{V}=$ Visitor/Camera person 